\title{
Identification of novel genes associated with dysregulation of $B$ cells in patients with primary Sjögren's syndrome
}

Jun Inamo ${ }^{1}$, Katsuya Suzuki ${ }^{1}$, Masaru Takeshita', Yoshiaki Kassai ${ }^{2}$, Maiko Takiguchi $^{2}$, Rina Kurisu², Yuumi Okuzono², Shinya Tasaki ${ }^{3,4}$, Akihiko Yoshimura ${ }^{5}$ and Tsutomu Takeuchi ${ }^{1 *}$ (D)

\begin{abstract}
Background: The aim of this study was to identify the molecular mechanism of dysregulation of B cell subpopulations of primary Sjögren's syndrome (pSS) at the transcriptome level.

Methods: We enrolled patients with pSS $(n=6)$ and healthy controls (HCS) $(n=6)$ in the discovery cohort using microarray and pSS $(n=14)$ and HCs $(n=12)$ in the validation cohort using quantitative PCR (qPCR). Peripheral B cells acquired from these subjects were separated by cell sorting into four subsets: $C^{2} 38^{-} \operatorname{lgD}{ }^{+}(B m 1), C D 38^{+} \operatorname{lgD}{ }^{+}$ (naive B cells), CD38 high $I g D^{+}$(pre-germinal centre B cells) and CD38 ${ }^{ \pm} \operatorname{lgD}{ }^{-}$(memory B cells). We performed differentially expressed gene (DEG) analysis and weighted gene co-expression network analysis (WGCNA).

Results: Expression of the long non-coding RNA LINC00487 was significantly upregulated in all B cell subsets, as was that of HLA and interferon (IFN) signature genes. Moreover, the normalized intensity value of LINC00487 significantly correlated with the disease activity score of all pSS B cell subsets. Studies of human B cell lines revealed that the expression of LINC00487 was strongly induced by IFNa. WGCNA revealed six gene clusters associated with the B cell subpopulation of pSS. Further, SOX4 was identified as an inter-module hub gene.
\end{abstract}

Conclusion: Our transcriptome analysis revealed key genes involved in the dysregulation of B cell subpopulations associated with pSS.

Trial registration: Not required.

Keywords: Primary Sjögren's syndrome, B cells, Long non-coding RNA, Transcriptome, Gene co-expression network

\section{Background}

Primary Sjögren's syndrome (pSS) is a systemic autoimmune disease characterized by exocrine gland dysfunction, which leads to dryness of the eyes and mouth [1]. Upregulation of the interferon (IFN) pathway [2] and dysregulation of $B$ cells play a critical role in the pathogenesis of pSS. First, the loss of B cell tolerance can lead to overproduction of anti-Sjögren's syndrome-

\footnotetext{
* Correspondence: tsutake@z5.keio.jp

'Division of Rheumatology, Department of Internal Medicine, Keio University School of Medicine, 35 Shinanomachi, Shinjuku-ku, Tokyo 160-8582, Japan Full list of author information is available at the end of the article
}

related antigen A (anti-SSA) autoantibodies. Autoreactive $B$ cells are important in the development of a clinical disease, because serum autoantibodies may precede the onset of dryness. Second, IFN-stimulated genes (ISGs) are upregulated in B cells in the peripheral blood as well as in the salivary gland lesions of patients with pSS [3, 4]. Third, IFNa promotes loss of tolerance and development of autoreactive B cells [5]. Genome-wide association studies identified single-nucleotide polymorphisms of ISGs that are associated with the risk of pSS $[6,7]$. Further, viral infection, which enhances the IFN pathway, may contribute to the pathogenesis of pSS [8]. 
Non-coding RNAs, which are emerging as critical regulators of signal transduction pathways, may be involved in the immune system. Non-protein-coding microRNAs are involved in the dysregulation of B cells [9] and in the upregulation of the IFN pathway in patients with pSS [10]. Long non-coding RNAs (lncRNAs) are involved in diverse regulatory functions, including the modulation of chromatin structure and post-transcriptional regulation affecting the stability of mRNAs and proteins [11]. Recent evidence indicates that IncRNAs, which have become the focus of studies on autoimmune diseases, may contribute to the pathogenesis of pSS through multiple signal transduction pathways [12]. For example, differentially expressed lncRNAs in the labial salivary glands of pSS patients were identified using microarray analysis [13]. In the multi-omics study of whole blood specimen, the ratio of B cell subpopulation within white blood cells was associated with the expression of pSS-associated signature genes, raising the necessity of in-depth investigation including lncRNAs about the link between dysregulated B cells and transcriptome [14].

Considering that the gene expression pattern is tissuespecific [15], it is better to focus on the target cell subpopulation to avoid noise from other cells in transcriptome analysis. However, we are unaware of studies that focus on the effects of dysregulation of transcriptomes, including lncRNAs, of B cells on the pathogenesis of pSS. Serum level of B cell-activating factor was associated with disease activity of pSS [16-18]. In addition, the distribution of peripheral B cell subsets is profoundly altered in patients with pSS [19]. Staining for IgD/CD38 is helpful for studying circulating B cell subsets, separating developmental stages from naive to memory B cells (Bm1 to $\mathrm{Bm} 5)$ [20]. Using this gating strategy, we previously reported that the proportion of pre-GC B cells $\left(\mathrm{IgD}^{+} / \mathrm{CD} 38^{\text {high }}\right)$ was higher in patients with pSS compared with healthy controls and associated with clinical traits, such as disease activity [19], indicating the importance of evaluating the involvement of each B cell subset in the pathogenesis of pSS.

To determine the role of the $\mathrm{B}$ cell subset in the pathogenesis of pSS, we investigated potentially significant genes according to their differential levels of expression and connectivity with other genes. Thus, the current study consists of two sections: differentially expressed gene (DEG) analysis and weighted gene coexpression network analysis (WGCNA). First, we describe the upregulation of the interferon signalling pathway and the differential expression of genes encoding human leucocyte antigen (HLA) molecules and LINC00487 in B cell subpopulations of patients with pSS compared with healthy controls (HCs). The expression levels of LINC00487 correlated with the disease activity of pSS and IFN signature genes, and LINC00487 was induced by IFN $\alpha$. Second, using WGCNA, we identified genes of co-expression networks specific to a B cell subset of patients with pSS, suggesting that aberrant molecular interactions in B cells contribute to the aetiology of pSS.

\section{Methods \\ Patients and controls}

The study protocol is shown in Fig. 1a. We enrolled patients with pSS $(n=6)$ and HCs $(n=6)$ matched for age and sex (Supplementary Table 1). The patients with pSS fulfilled the 2002 American-European criteria for SS [21] and the 2012 American College of Rheumatology classification criteria for SS [22]. All patients were female and had a symptom of dryness, anti-SSA antibodies and biopsy-proven sialadenitis. The patients had not received immunosuppressive therapy, and the $\mathrm{HCs}$ did not have immunological disorders. Clinical and serological information of patients with pSS were collected from their medical records. The disease activity of pSS was assessed according to the guidelines of the European League against Rheumatism and the Primary Sjögren's Syndrome Disease Activity Index (ESSDAI) [23, 24]. The distribution of ESSDAI domain and clinical information of individual patients were described in Supplementary Table 17 and Supplementary Table 18.

This study was performed in accordance with relevant guidelines and regulations. The Ethics Committee of Keio University School of Medicine approved this study (IRB No. 20110258), and written informed consent was obtained from each subject before blood collection.

\section{Cell sorting}

Peripheral blood mononuclear cells from patients with pSS and HCs were separated using gradient centrifugation with Lymphoprep (Axis-Shield; Oslo, Norway). Gating strategy was shown in Supplementary Figure 1. Peripheral $\mathrm{CD} 19^{+} \mathrm{B}$ cells were prepared with anti-CD19 antibody-coated microbeads (Miltenyi Biotec). As previously reported [19], the peripheral $\mathrm{CD} 19^{+} \mathrm{B}$ cells were incubated with anti-IgD and CD38 antibodies for fluorescence-activated cell sorting (FACS) analysis (FACSAria III flow cytometer, BD Biosciences). We defined subsets of $\mathrm{B}$ cells as follows: $\mathrm{Bm} 1$ cells, $\mathrm{CD}^{2} 8^{-} \mathrm{IgD}{ }^{+}$; naive $\mathrm{B}$ cells, $\mathrm{CD} 8^{+} \mathrm{IgD}^{+}$; pre-germinal centre (pre-GC) B cells, CD $38^{\text {high }} \mathrm{IgD}^{+}$; and memory B cells, CD $38^{ \pm} \mathrm{IgD}^{-}$.

\section{DEG analysis}

Total RNA was extracted from B cell subsets and transcribed into cDNA using NucleoSpin RNA (Macherey Nagel) and ReverTra Ace qPCR RT Master Mix (Toyobo). Gene expression was measured using the $\mathrm{Hu}$ man Genome U133 Plus 2.0 Array (Affymetrix). We 


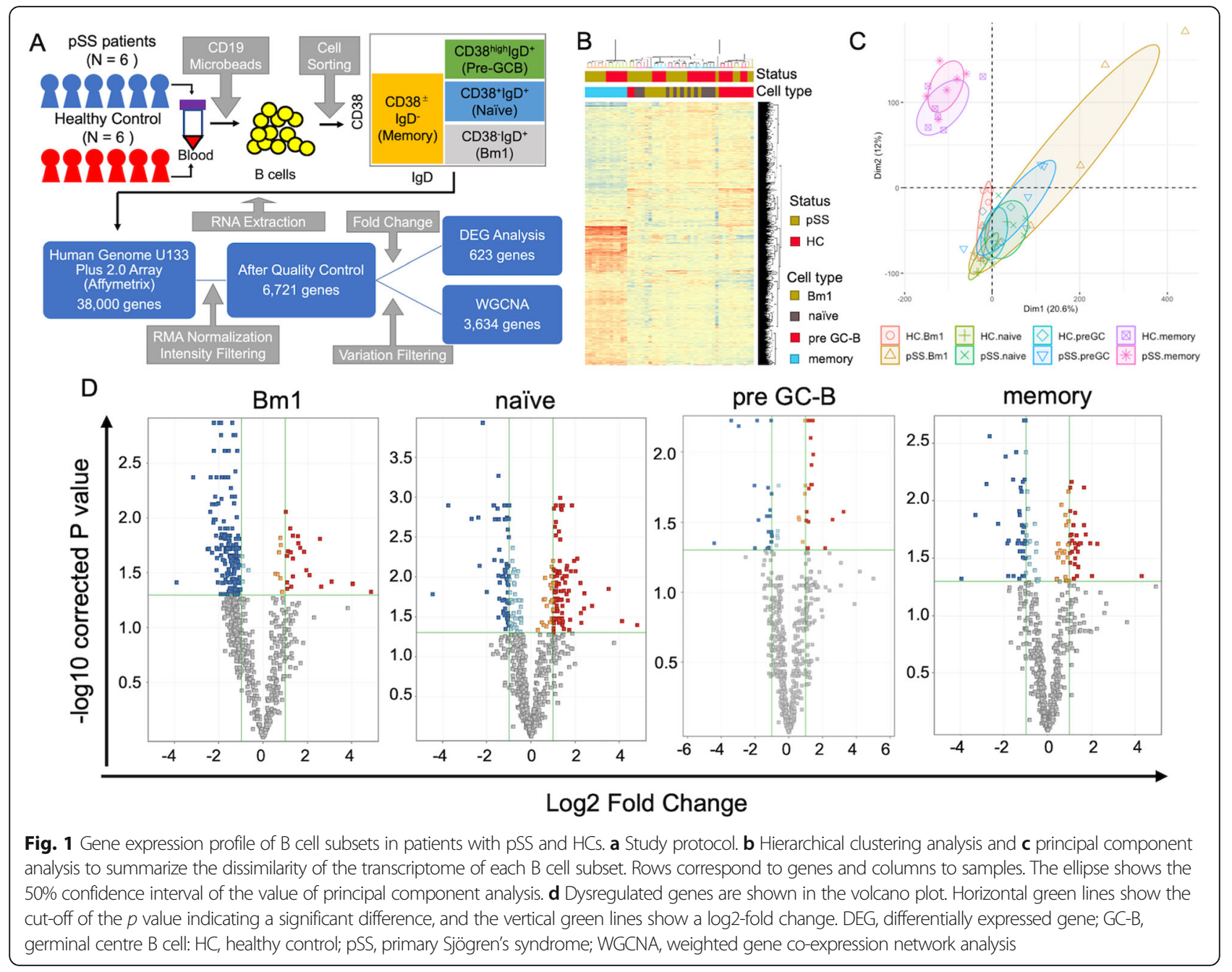

applied percentile shift normalization to the raw signal data acquired from a microarray and annotated each probe with its gene symbol using the GeneSpring software (Agilent Technologies). Probes with interquartile ranges in the lowest $20 \%$ were excluded. We next selected probes with $>2.0$ changes for pSS vs $\mathrm{HCs}$ in any one B cell subset to identify DEGs. We controlled for the false discovery rate using the Bonferroni multiple testing-corrected $p$ value $<0.05$. To functionally characterize DEGs identified in each B cell subpopulation from microarray analysis, we performed a pathway analysis using Enrichr's plugin [25] BioPlanet [26]. The BioPlanet database incorporates more than 1500 human pathways sourced from publicly available, manually $\mathrm{cu}-$ rated sources. In a pathway analysis, $p$ value was adjusted using the Benjamini-Hochberg method for correction for multiple hypotheses testing.

\section{WGCNA}

To explore novel gene co-expression networks and common hub genes, we created another gene set. In order to select genes that are steadily expressed in each B cell subpopulation of pSS and HCs (totally, 8 subpopulations; $\mathrm{Bm} 1$, naive, pre-GC and memory $\mathrm{B}$ cells of pSS, and Bm1, naive, pre-GC and memory B cells of $\mathrm{HCs}$ ), we removed genes with coefficients of variation greater than 0.3 according to each subpopulation after intensity filtering and applied them to the WGCNA R package [27] following the package's tutorial. Briefly, we applied step-by-step construction of the gene network and identification of modules to fileted gene expression dataset. First, we chose the proper soft-thresholding power based on the criterion of approximate scale-free topology. After calculating the adjacencies with selected softthresholding power, we transformed the adjacency into a topological overlap matrix and calculate the corresponding dissimilarity. Then, we used hierarchical clustering to produce a hierarchical clustering tree of genes. Module identification amounts to the identification of individual branches; we cut the branches off the dendrogram with setting minimum module size 30 . As a result, 6 and 5 modules were generated in B cells of 
patients with pSS and HCs, respectively. Further, to annotate and estimate the regulatory mechanism of a coexpressed gene network, we investigated the pathways, upstream regulators and functions associated with modules by using Ingenuity Pathway Analysis (IPA).

\section{Cell culture and real-time quantitative PCR}

B cell lines (Ramos, CCRF, U266B1) (each $1.0 \times 10^{5} / \mathrm{mL}$ ) in RPMI 1640 medium (American Type Culture Collection) supplemented with $10 \%$ foetal calf serum were treated with different concentrations of IFN $\alpha$, IFN $\gamma$ and TNF $\alpha$ for $48 \mathrm{~h}$. We next isolated total RNA that was transcribed into cDNAs using NucleoSpin RNA (Macherey Nagel) and ReverTra Ace qPCR RT Master Mix (Toyobo). qPCR was performed using PowerUp SYBR Green Master Mix (Thermo Fisher Scientific). The levels of total cellular RNA were similar amongst the cell lines. At least two biological repeats were performed for each experiment. The levels of GAPDH mRNA were used to adjust the values of target transcripts determined using $\mathrm{qPCR}$ analyses. We validated gene expression levels in $\mathrm{CD} 19^{+} \mathrm{B}$ cells of patients with pSS $(n=14)$ and HCs $(n=12)$ (Supplementary Table 2) using quantitative PCR (qPCR) analysis. The sequence of primers for qPCR analysis was described in Supplementary Table 3.

\section{Statistics}

Continuous values are shown as the median and range of values. Differences between the groups were analysed using the Mann-Whitney test for continuous variables. Correlations were analysed using Spearman's correlation coefficient, unless otherwise noted. $p<0.05$ indicates a significant difference. Statistical tests were conducted using R software version 3.5.2.

\section{Results}

Identification of gene signatures in B cell subsets of pSS

First, we visualized the gene expression profile after intensity filtration. Using principal component and hierarchical clustering analyses, we found that clinical status, namely pSS or $\mathrm{HCs}$, had less influence on phenotype than the type of B cell subset (Fig. 1b, c). Memory B cells, in particular, had a distinct expression pattern compared with those of other cell types.

To determine the gene signatures of B cell subset of patients with pSS, we identified DEGs and performed pathway analyses. Gene selection yielded 623 significant genes (Supplementary Table 4). Analysis of changes in the expression indicated that 23, 92, 18 and 32 genes were upregulated in $\mathrm{Bm} 1$, naive, pre-GC and memory $\mathrm{B}$ cell subsets, respectively (Fig. 1d).

Consistent with previous reports, ISGs were upregulated in B cells of pSS compared with those of HCs
(Fig. 2a-d). Further, HLA genes were similarly upregulated. Pathway analysis demonstrated that the interferon pathway was the most significantly upregulated pathway in all of B cell subsets (Fig. 2e-h). In contrast, regarding downregulated genes, there was no enriched pathway. We found that a lncRNA, LINC00487, was amongst the 15 probes significantly upregulated in all B cell subsets of pSS compared with those of HCs (Supplementary Table 5). The fold changes of LINC00487 expression were as follows: $\mathrm{Bm} 1,8.4 \quad(p=0.038)$; naive, 11.3 $(p=0.014)$; pre-GC, $8.5(p=0.089)$; and memory, 6.37 $(p=0.078) \quad$ (Fig. 2a-d). There were no significant differences amongst the $B$ cell subsets examined for HCs or pSS patients (Supplementary Figure 2).

\section{Expression of LINC00487 correlates with IFN signature genes and disease activity score of pSS}

To determine the characteristics of LINC00487, we identified the top three genes that correlated with LINC00487 amongst all of the subsets (interferon-induced protein 44 like [IFI44L], interferon-induced protein with tetratricopeptide repeats 3 [IFIT3] and interferoninduced protein 44 [IFI44], in the order of low $p$ value). All three genes were known ISGs. Expression of LINC00487 and these ISGs were significantly correlated in more subsets of pSS compared with HCs (9 vs 1 subset, respectively) (Fig. 3a, b). Further, the clinical disease activity score was correlated with the expression level of LINC00487 in all pSS B cell subsets: Bm1, $r=0.98$ $(p<0.01)$; naive, $r=0.93(p<0.01)$; pre-GC, $r=0.84$ $(p=0.03)$; and memory, $r=0.93(p<0.01)$ (Fig. 3c). However, it should be noted that only patients with low ESSDAI scores $(<5)$ were included in the current cohort.

Using a validation cohort, we tried to conduct a qPCR analysis using each $\mathrm{B}$ cell subset. However, because LINC00487 was expressed at a very low level in HCs, we could not sort enough each B cell subset from them to detect the expression of LINC00487. Therefore, considering that upregulation of LINCO0487 was common in all $\mathrm{B}$ cell subsets in microarray cohort, we used a bulk of $\mathrm{CD} 19^{+} \mathrm{B}$ cells in the validation cohort. As a result, the expression of LINC00487 in CD19 ${ }^{+}$B cells of patients with pSS was significantly upregulated compared with HCs $(p=0.035)$ (Fig. 3d). The same as Fig. 3a and b, the expression of LINC00487 was correlated with IFI44L with lower $p$ value in patients with pSS $(p<0.001)$ (Fig. 3e) compared with that of HCs $(p=0.04)$ (Fig. 3f). To confirm the induction of LINC00487 in response to IFN $\alpha$, we used three types of B cell lines and measured the LINC00487 expression using qPCR. As with IFI44L, LINC00487 was strongly induced by treatment with IFN $\alpha$ (Fig. 3e, f), unlike TNF $\alpha$ and IFN $\gamma$ (Supplementary Figure $3 \mathrm{~A}$ ), suggesting that IFN $\alpha$ is an upstream regulator of LINC00487. Compared with clinical features, the 

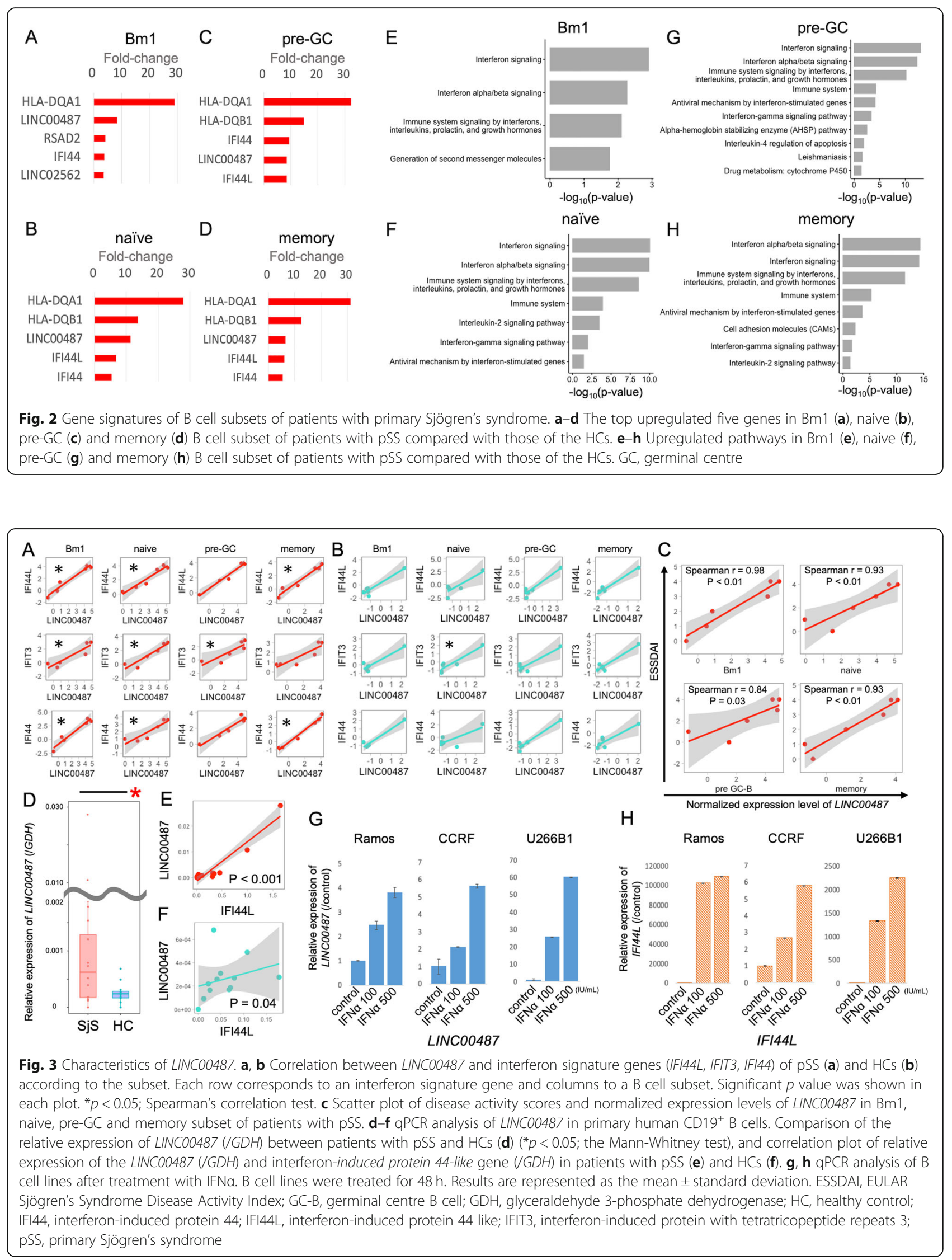
expression of LINC00487 tended to be higher in patients with higher serum IgG levels and lower lymphocyte counts which were characteristic for pSS (Supplementary Figure 3B).

\section{Co-expression gene networks constructed for each B cell subset}

To identify transcriptional networks in the B cells of patients with pSS, we performed WGCNA. WGCNA can identify novel gene interactions which might be missed in the DEG analysis, because WGCNA is based on correlations of gene expression [25].

After selection, we identified a new set comprising 3634 genes (Supplementary Tables 6-7). In the principal component analysis using these gene sets, $\mathrm{Bm} 1$ and naive $B$ cells in pSS tended to be closer to the expression patterns of a more highly differentiated subset compared to those in $\mathrm{HCs}$, i.e. $\mathrm{Bm} 1$ to naive and naive to pre-GC (Fig. 4a). WGCNA identified 6 modules in pSS and an association between cell subsets and modules (Fig. 4b). For example, the yellow and brown modules are associated with the pre-GC B cell subset, whereas the turquoise module is associated with the memory B cell subset. The grey module of pSS was enriched in Bm1/ naive $B$ cell subsets with various ranges of eigengenes. In the analysis to detect an association between modules and clinical traits, the grey module significantly correlated with the clinical disease activity score (Fig. 4c). Similarly, another five modules were identified in HCs (Supplementary Figure 4A). The module pairs with the greatest number of genes in each pSS module overlapping with either $\mathrm{HC}$ modules were blue (pSS)-turquoise $(\mathrm{HC})$, brown (pSS)-brown (HC), grey (pSS)-blue (HC), green (pSS)-turquoise ( $\mathrm{HC})$, yellow (pSS)-blue $(\mathrm{HC})$ and turquoise (pSS)-turquoise (HC) (Supplementary Figure 5). All of the above pairs overlapped more than half of the gene list, suggesting that the overall picture of the B cell modules in pSS and the B cell modules in HCs was similar.

To extract the characteristics of pSS modules, we performed a functional analysis using Ingenuity Pathway Analysis (IPA). The cluster of enriched pathway, upstream regulator and disease/biological functions in modules of pSS were distinguished from those of $\mathrm{HCs}$ (Fig. 5a and Supplementary Tables 8-13). The top five most significantly enriched pathways, regulators and disease/biological functions in modules of pSS are shown in Fig. 5b. In the upstream regulator analysis, transcriptional regulators such as Sry-related high-mobility group box (Sox) family such as SOX4 were identified in the yellow and blue modules of pSS. Focusing on specificity (Supplementary Figure 6 and Supplementary Tables 1416), mir-21-5p was identified as the top significant regulator in the yellow module of pSS. To search for hub genes, we described a co-expressed gene network using top highly interconnected genes defined by a topological overlap in each module (Fig. 5c). Several genes bridged inter-modules, namely hub genes, such as SOX4. SOX4 was not identified in the co-expression network of HCs (Supplementary Figure 4B), suggesting its involvement in the dysregulation of B cells in pSS.

\section{Discussion}

In the present study, we conducted a transcriptome analysis of B cell subpopulations. First, we found that the expression of LINC00487 was upregulated in B cell subsets derived from patients with pSS. Further, LINC00487 expression significantly correlated with disease activity and was induced by IFN $\alpha$ stimulation. Second, using WGCNA, we identified several key networks and hub genes in the B cells of patients with pSS.

IFN signalling is a central component of the pathogenesis of pSS [28]. Type 1 IFN signalling plays a crucial role in the development of autoreactive B cells in a mouse model of autoimmune disease [5]. Principal component analysis using gene sets for WGCNA demonstrated that $\mathrm{Bm} 1 /$ naive $\mathrm{B}$ cells of pSS were clearly distinguished from those of $\mathrm{HCs}$ and showed a trend of expression patterns closer to the more highly differentiated subset. These results suggest that molecular dysfunction, such as disruption of peripheral tolerance, might start at an early stage in the maturation of B cells. Indeed, the frequencies of naive B cells expressing autoreactive antibodies are significantly increased in patients with pSS [29].

Interestingly, expression of the HLA class II gene was upregulated. GWAS studies of pSS found associations of $H L A-D Q A 1$ and $H L A-D Q B 1$ loci $[6,7]$. Further, IFNa induces the expression of $H L A-D Q A 1$ [30]. In patients with pSS, $H L A-D Q A 1$ and $H L A-D Q B 1$ alleles are associated with higher concentrations of anti-SSA and SSB antibodies [31]. These findings suggest that aberrant interactions amongst IFN signalling and HLA class II genes may trigger the breakdown of B cell tolerance, leading to the development of pSS.

LINC00487 was upregulated in all B cell subsets of pSS, and its expression significantly correlated with the disease activity scores of pSS and ISGs, although the ESSDAI score of our patients skewed toward the low end. Moreover, we found that IFN $\alpha$ was an upstream regulator of LINC00487 in B cells. The sequence of LINC00487, which belongs to the class of long intergenic non-coding RNAs and resides on human chromosome 2 , is atypically long ( $>40,000$ bases). Although LINC00487 is one of the hub genes in the normal development of human B cells [32], many of its properties, including its function, are unexplained. Further, there is no ortholog or paralog of this gene in species other than 


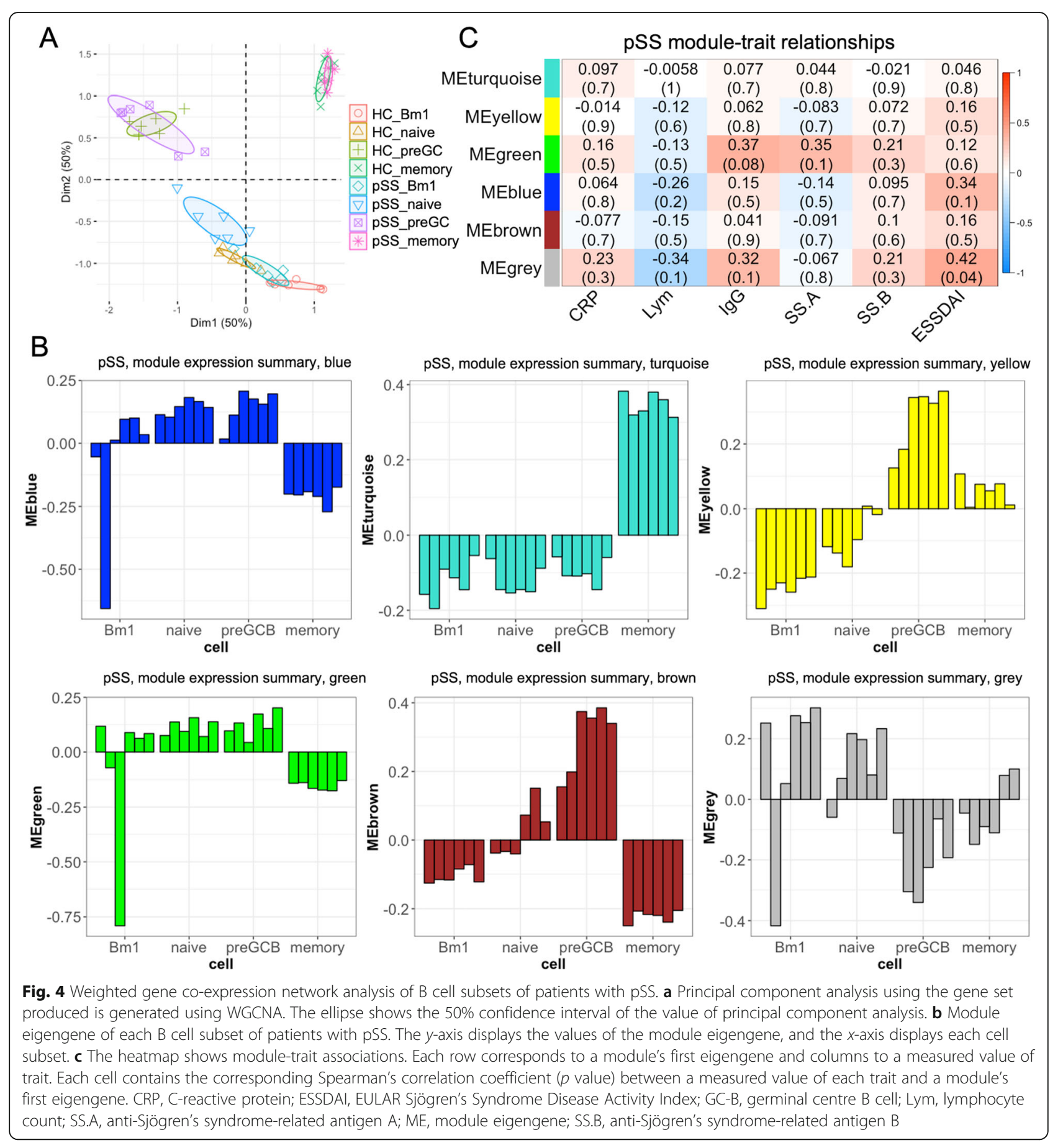

humans that may provide clues to its function in human cells. However, according to AceView, one of three transcriptional variants derived from LINC00487 has the potential to encode a protein in silico prediction [33].

Four genomic locations are considered candidate enhancers of LINC00487 transcription. The targets of the regulators of LINC00487 overlap with those of other ISGs [34]. Moreover, referring to the public transcriptome database of microarray analyses of healthy humans, expression of LINC00487 is higher specifically in centroblasts and centrocytes of the germinal centre [35]. IFN $\alpha$ promotes the autoreactivity of $\mathrm{B}$ cells via germinal centre pathways [5]. Further, LINC00487 expression is upregulated in the subgroup of diffuse large B cell lymphoma with molecular characteristics of germinal centre B cells, compared with other subgroups, and is associated with the efficacy of B cell depletion therapy [36]. Therefore, our study suggests that upregulation of LINC00487 


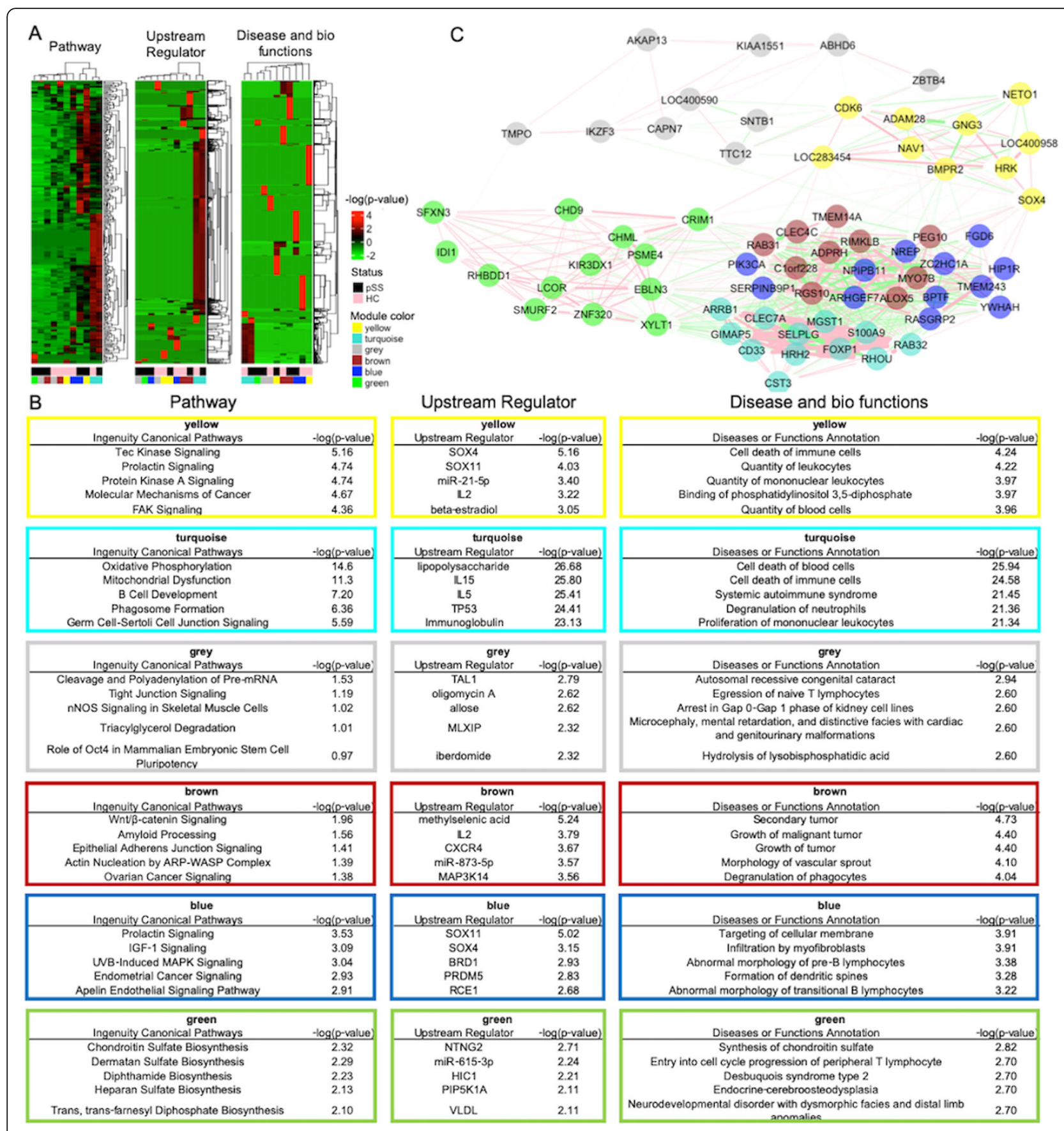

Fig. 5 Gene network characteristics and hub genes in the gene networks of B cells of pSS. a Hierarchical clustering analysis with a heatmap of enriched pathways (left), upstream regulators (middle) and disease/functions (right). b Top five significantly enriched canonical pathways (left), upstream regulators (middle) and disease and biological functions (right) in the modules of pSS. The colour of each frame corresponds to a module colour. c Associations of intra- and inter-modular hub genes in patients with pSS. Node colour corresponds to module colour. The pink-coded edge represents the correlation, and the green-coded edge represents an inverse correlation. The width of the edge reflects the absolute weight of a correlation

expression in all $\mathrm{B}$ cell subsets may reflect or regulate the enrichment of a germinal centre-like reaction by IFN $\alpha$ from an early stage of B cell development, leading to $\mathrm{B}$ cell autoreactivity in patients with pSS.

Gene co-expression analysis revealed an aberrant network in B cell subpopulations. The top significant upstream regulator of the grey module of pSS, which was associated with clinical disease activity score and enriched in the early stage of B cell development in pSS, was the gene encoding $\mathrm{T}$ cell acute lymphocytic leukaemia protein 1 (TAL1). B cell development is stringently controlled by stage-specific transcription factors. The 
transcription factor TAL1 regulates genes such as IKAROS family zinc finger 3 (IKZF3), which is one of the hub genes in the grey module of pSS (Fig. 5c). IKZF3 is a lineage-specific transcription factor that is important in the regulation of $\mathrm{B}$ cell proliferation and development [37].

In the pre-GC B cell-associated module of pSS, SOX4 was identified as an upstream regulator and a hub gene. In mice, SOX4 regulates the differentiation of early-stage B cells by activating the expression of Rag1 and Rag2 [38]. Further, SOX4 contributes to the formation of ectopic lymphoid-like structures via promoting CXCL13, which is a ligand of CXCR5 on naive B cells and critical for migration into the light zone of germinal centre undergoing somatic hypermutation, production from PD- $1{ }^{\text {hi }}$ CXCR $5^{-} \mathrm{CD} 4^{+} \mathrm{T}$ cells [39]. Additionally, in proteome analysis, CXCL13 positively correlates with the disease activity score and serum IgG levels of patients with pSS [19]. Further study is needed to explore the role of SOX4 in mature B cells.

Further, we identified miR-21 as an upstream regulator of the yellow module of pSS. Although miR-21 is upregulated in peripheral blood mononuclear cells of pSS [40], the present study is the first to propose its involvement in pSS via B cell dysregulation. Interestingly, miR21 regulates the immune response of memory $\mathrm{T}$ cells via induction of transcription networks, such as SOX4 [41], implying that the interaction between miR-21 and SOX4 may also affect the function of B cells.

pSS is an enormously heterogeneous disease from the molecular point of view. In WGCNA, despite filtering out genes with high variation in each cell subpopulation, module expression showed the strong variation amongst samples in the same group, in particular Bm1 subset (Fig. 4b). One of the reasons for the high variability seen in Bm1 subset may be related to the fact that some cells with the $\mathrm{CD}^{-} 8^{-} \mathrm{IgD}^{+}$express CD27 [20]. Namely, the $\mathrm{CD}^{-} 8^{-} \mathrm{IgD}^{+} \mathrm{B}$ cell subpopulation includes both naive $\mathrm{Bm} 1$ cells and $\mathrm{IgD}^{+}$memory $\mathrm{B}$ cells. However, the finding in the current study suggested that there might be different regulatory mechanisms within each subgroup, although it was information that could not be analysed due to the limited sample size.

The current study suffers from several limitations. First, patients in our cohort have low disease activity/severity. Because there were few patients with high disease activity before immunosuppressive treatment, it was difficult to include such patients in this study. Therefore, the correlation between the expression of LINC00487 and disease activity score is needed to be validated in another cohort including patients with high ESSDAI scores. Second, healthy controls were younger than the pSS patients, and this could be a confounding variable. Third, the sample size was limited. To overcome the limitation about the small sample size, we validated by qPCR using another cohort, supporting the results derived from microarray analysis. However, regarding WGCNA, we could not include subjects enough to replicate by qPCR.

\section{Conclusions}

Our focus on B cell subpopulation using a multi-level approach employing the analysis of DEGs and WGCNA identified significant genes and networks as novel players in the pathogenesis of pSS. To confirm our results, further study is needed.

\section{Supplementary information}

Supplementary information accompanies this paper at https://doi.org/10. 1186/s13075-020-02248-2.

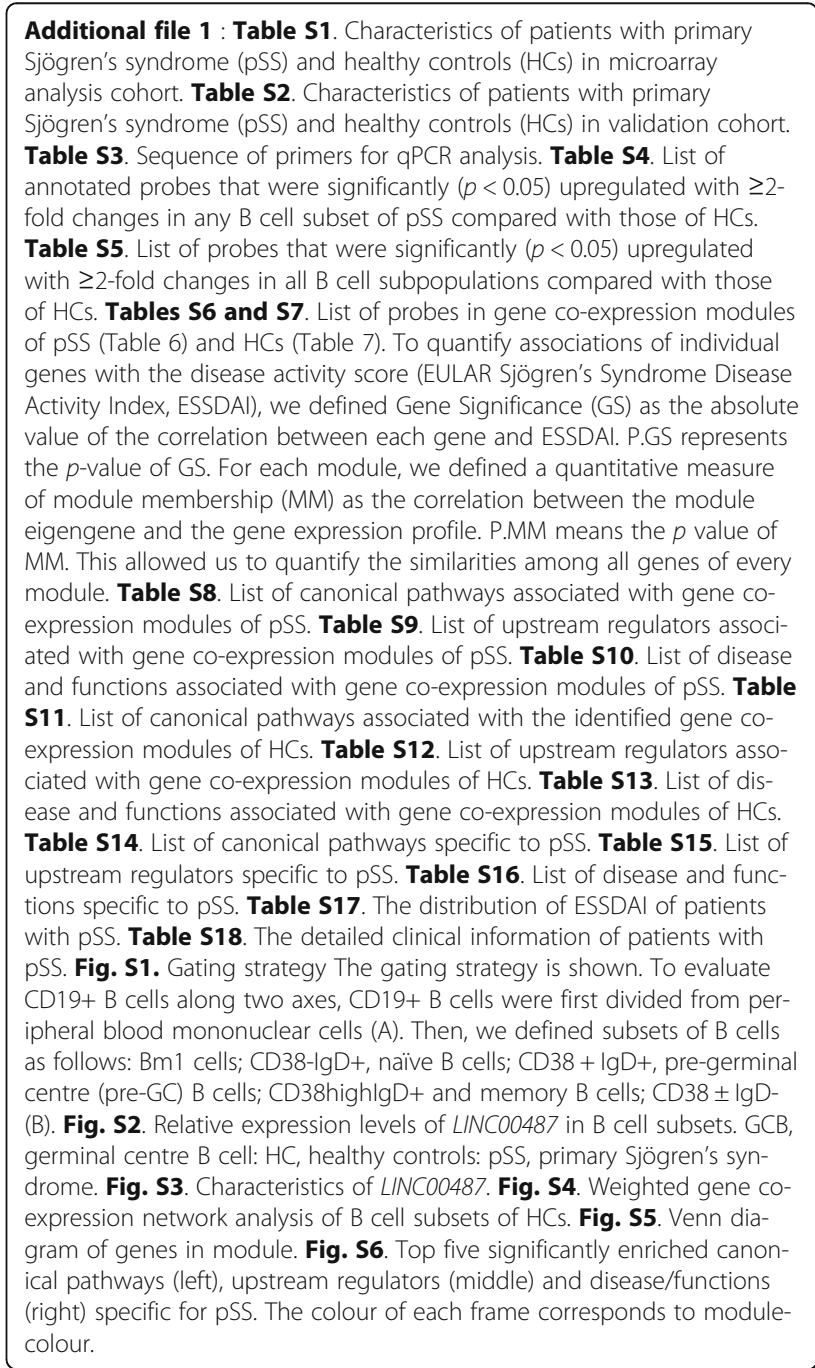

\section{Abbreviations}

ESSDAl: EULAR Sjögren's Syndrome Disease Activity Index;

FACS: Fluorescence-activated cell sorting; GC-B: Germinal centre B cell; HC: Healthy control; HLA: Human leucocyte antigen; IFN: Interferon; IKZF3: IKAROS family zinc finger 3; ISGs: Interferon-stimulated genes; 
IncRNA: Long non-coding RNA; pSS: Primary Sjögren's syndrome; TAL1: T cell acute lymphocytic leukaemia protein 1; WGNCA: Weighted gene coexpression network analysis

\section{Acknowledgements}

This work was supported by Takeda Pharmaceutical Company Limited, Kanagawa, Japan (grant number 04-078-0067), and Keio University (grant number 04-013-0188).

We thank Ms. Harumi Kondo, Ms. Mayumi Ota, Ms. Yoshiko Yogiashi, Ms. Yuki Otomo and Mr. Fumitsugu Yamane for helping with the experiments and Digital Medical Communications Corp. for proofreading the sentences for English proofreading.

\section{Authors' contributions}

Conceptualization: Jl, KS, MTake, YK, YO, MTaki, RK and ST. Funding acquisition: KS and TT. Data acquisition: YK, MTaki and RK. Formal analysis: Jl, YO and ST. Supervision: KS. Writing and original draft preparation: Jl. Writing review and editing: $\mathrm{KS}, \mathrm{AY}$ and $\mathrm{TT}$. The authors read and approved the final manuscript.

\section{Funding}

This study was funded by Keio University (grunt number: 04-013-0188) and Takeda Pharmaceutical Company Limited (grunt number: 04-078-0067).

\section{Availability of data and materials}

The transcriptome data are available at the GEO database. The accession code is GSE135809. All custom computer codes in the generation or processing of the described data are available upon reasonable request. Supplementary Tables 4-18 and R script for WGCNA are deposited in figshare (https://figshare.com/articles/Supplementary_Table_4-14/11683959).

\section{Ethics approval and consent to participate}

Ethics approval was obtained from the Institutional Review Board of Keio University School of Medicine (IRB No. 20110258). Consent to participate was obtained from all subjects in the current study before the blood specimen was collected.

\section{Consent for publication}

Not applicable.

\section{Competing interests}

YK, YO, MTaki and RK are employed by Takeda Pharmaceutical Company Limited. S.T. was employed by Takeda Pharmaceutical Company Limited. KS has received research grants from Eisai, Bristol-Myers Squibb, Kissei Pharmaceutical and Daiichi Sankyo; speaking fees from Abbie Japan, Astellas Pharma, Bristol-Myers Squibb, Chugai Pharmaceutical, Eisai, Fuji Film Limited, Janssen Pharmaceutical, Kissei Pharmaceutical, Mitsubishi Tanabe Pharmaceutical, Pfizer Japan, Shionogi, Takeda Pharmaceutical and UCB Japan; and consulting fees from Abbie and Pfizer Japan. AY has received speaking fees from Chugai Pharmaceutical, Mitsubishi Tanabe Pharmaceutical, Pfizer Japan, Ono Pharmaceutical, Maruho and Novartis, and consulting fees from GSK Japan. $T$ Thas received research grants from Astellas Pharma Inc., Bristol-Myers KK, Chugai Pharmaceutical Co. Ltd., Daiichi Sankyo Co. Ltd., Takeda Pharmaceutical Co. Ltd., Teijin Pharma Ltd., AbbVie GK, Asahikasei Pharma Corp, Mitsubishi Tanabe Pharma Co, Pfizer Japan Inc., Taisho Toyama Pharmaceutical Co. Ltd., Eisai Co. Ltd., AYUMI Pharmaceutical Corporation and Nipponkayaku Co. Ltd.; speaking fees from AbbVie GK, Bristol-Myers KK, Chugai Pharmaceutical Co. Ltd., Mitsubishi Tanabe Pharma Co, Pfizer Japan Inc., Astellas Pharma Inc. and Diaichi Sankyo Co. Ltd.; and consultant fees from Astra Zeneca KK, Eli Lilly Japan KK, Novartis Pharma KK, Mitsubishi Tanabe Pharma Co, Abbivie GK, Nipponkayaku Co. Ltd., Janssen Pharmaceutical KK, Astellas Pharma Inc. and Taiho Pharmaceutical Co. Ltd. JI and MT declare no potential conflict of interest.

\section{Author details}

'Division of Rheumatology, Department of Internal Medicine, Keio University School of Medicine, 35 Shinanomachi, Shinjuku-ku, Tokyo 160-8582, Japan. ${ }^{2}$ Immunology Unit, Pharmaceutical Research Division, Takeda Pharmaceutical Company Limited, Kanagawa, Japan. ${ }^{3}$ Integrated Technology Research Laboratories, Pharmaceutical Research Division, Takeda Pharmaceutical Company Limited, Kanagawa, Japan. ${ }^{4}$ Rush Alzheimer's Disease Center, Rush
University Medical Center, Chicago, USA. ${ }^{5}$ Department of Microbiology and Immunology, Keio University School of Medicine, Tokyo, Japan.

Received: 29 April 2020 Accepted: 15 June 2020

Published online: 22 June 2020

\section{References}

1. Qin B, Wang J, Yang Z, et al. Epidemiology of primary Sjögren's syndrome: a systematic review and meta-analysis. Ann Rheum Dis. 2015;74:1983-9.

2. Muskardin TLW, Niewold TB. Type I interferon in rheumatic diseases. Nat Rev Rheumatol. 2018;14:214-28.

3. Imgenberg-Kreuz J, Sandling JK, Björk A, et al. Transcription profiling of peripheral B cells in antibody-positive primary Sjögren's syndrome reveals upregulated expression of CX3CR1 and a type I and type II interferon signature. Scand J Immunol. 2018;87:e12662.

4. Devauchelle-Pensec V, Cagnard N, Pers JO, et al. Gene expression profile in the salivary glands of primary Sjögren's syndrome patients before and after treatment with rituximab. Arthritis Rheum. 2010;62:2262-71.

5. Domeier PP, Chodisetti SB, Schell SL, et al. B-cell-intrinsic type 1 interferon signaling is crucial for loss of tolerance and the development of autoreactive B cells. Cell Rep. 2018;24:406-18.

6. Lessard CJ, Li H, Adrianto I, et al. Variants at multiple loci implicated in both innate and adaptive immune responses are associated with Sjögren's syndrome. Nat Genet. 2013;45:1284-92.

7. Li Y, Zhang K, Chen H, et al. A genome-wide association study in Han Chinese identifies a susceptibility locus for primary Sjögren's syndrome at 7q11.23. Nat Genet. 2013;45:1361-5.

8. Triantafyllopoulou A, Moutsopoulos H. Persistent viral infection in primary Sjögren's syndrome: review and perspectives. Clin Rev Allergy Immunol. 2007:32:210-4.

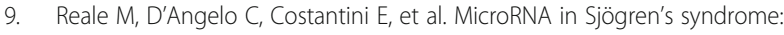
their potential roles in pathogenesis and diagnosis. J Immunol Res. 2018; 7510174.

10. Wang-Renault SF, Boudaoud S, Nocturne G, et al. Deregulation of microRNA expression in purified $\mathrm{T}$ and $\mathrm{B}$ lymphocytes from patients with primary Sjögren's syndrome. Ann Rheum Dis. 2018;77:133-40.

11. Tang $Y$, Zhou T, Yu X. The role of long non-coding RNAs in rheumatic diseases. Nat Rev Rheumatol. 2017;13:657-69.

12. Sandhya P, Joshi K, Scaria V. Long noncoding RNAs could be potential key players in the pathophysiology of Sjögren's syndrome. Int J Rheum Dis. 2015;18:898-905.

13. Shi H, Cao N, Pu Y, et al. Long non-coding RNA expression profile in minor salivary gland of primary Sjögren's syndrome. Arthritis Res Ther. 2016;18:109.

14. Tasaki S, Suzuki K, Nishikawa A, et al. Multiomic disease signatures converge to cytotoxic CD8 T cells in primary Sjögren's syndrome. Ann Rheum Dis. 2017;76:1458-66.

15. GTEx Consortium. Human genomics. The Genotype-Tissue Expression (GTEx) pilot analysis: multitissue gene regulation in humans. Science. 2015;348: 648-60.

16. Nishikawa A, Suzuki K, Kassai $Y$, et al. Identification of definitive serum biomarkers associated with disease activity in primary Sjögren's syndrome. Arthritis Res Ther. 2016;18:106.

17. Lee SJ, Oh HJ, Choi BY, et al. Serum 25-hydroxyvitamin D3 and BAFF levels are associated with disease activity in primary Sjogren's syndrome. J Immunol Res. 2016;2016:5781070.

18. James K, Chipeta C, Parker A, et al. B-cell activity markers are associated with different disease activity domains in primary Sjögren's syndrome. Rheumatology (Oxford). 2018:57:1222-7.

19. Ishioka-Takei E, Yoshimoto K, Suzuki K, et al. Increased proportion of a CD38 ${ }^{\text {high }} \operatorname{lgD}+\mathrm{B}$ cell subset in peripheral blood is associated with clinical and immunological features in patients with primary Sjögren's syndrome. Clin Immunol. 2018;187:85-91.

20. Bohnhorst J $\varnothing$, Bjørgan MB, Thoen JE, et al. Bm1-Bm5 classification of peripheral blood $B$ cells reveals circulating germinal center founder cells in healthy individuals and disturbance in the B cell subpopulations in patients with primary Sjögren's syndrome. J Clin Invest. 2005;115:3205-16.

21. Vitali C, Bombardieri S, Jonsson R, et al. Classification criteria for Sjögren's syndrome: a revised version of the European criteria proposed by the American-European Consensus Group. Ann Rheum Dis. 2002;61:554-8.

22. Shiboski SC, Shiboski CH, Criswell L, et al. American College of Rheumatology classification criteria for Sjögren's syndrome: a data-driven, 
expert consensus approach in the Sjögren's International Collaborative Clinical Alliance Cohort. Arthritis Care Res (Hoboken). 2012;64:475-87.

23. Seror R, Ravaud P, Bowman SJ, et al. EULAR Sjögren's syndrome disease activity index: development of a consensus systemic disease activity index for primary Sjögren's syndrome. Ann Rheum Dis. 2010;69:1103-9.

24. Seror R, Bowman SJ, Brito-Zeron P, et al. EULAR Sjögren's syndrome disease activity index (ESSDAI): a user guide. RMD Open. 2015;1:e000022.

25. Chen EY, Tan CM, Kou Y, et al. Enrichr: interactive and collaborative HTML5 gene list enrichment analysis tool. BMC Bioinformatics. 2013;14:128.

26. Huang R, Grishagin I, Wang Y, et al. The NCATS BioPlanet - an integrated platform for exploring the universe of cellular signaling pathways for toxicology, systems biology, and chemical genomics. Front Pharmacol. 2019;10:445

27. Langfelder P, Horvath S. WGCNA: an R package for weighted correlation network analysis. BMC Bioinformatics. 2008;9:559.

28. Nocturne G, Mariette X. B cells in the pathogenesis of primary Sjögren's syndrome. Nat Rev Rheumatol. 2018;14:133-45.

29. Glauzy S, Sng J, Bannock JM, et al. Defective early B cell tolerance checkpoints in Sjögren's syndrome patients. Arthritis Rheumatol. 2017;69: 2203-8.

30. Harris PE, Malanga D, Liu Z, et al. Effect of interferon alpha on MHC class II gene expression in ex vivo human islet tissue. Biochim Biophys Acta. 1762; 2006:627-35.

31. Harley JB, Reichlin M, Arnett FC, et al. Gene interaction at HLA-DQ enhances autoantibody production in primary Sjögren's syndrome. Science. 1986;232: $1145-7$.

32. Petri A, Dybkær K, Bøgsted $M$, et al. Long noncoding RNA expression during human B-cell development. PLoS One. 2015;10:e0138236.

33. Thierry-Mieg D, Thierry-Mieg J. AceView: a comprehensive cDNA-supported gene and transcripts annotation. Genome Biol. 2006;7(Suppl 1):S12.1-14.

34. Fishilevich S, Nudel R, Rappaport N, et al. GeneHancer: genome-wide integration of enhancers and target genes in GeneCards. Database (Oxford). 2017:2017:bax028

35. McCall MN, Uppal K, Jaffee HA, et al. The gene expression barcode: leveraging public data repositories to begin cataloging the human and murine transcriptomes. Nucleic Acids Res. 2011;39:D1011-5

36. Jiayu Yu, Alyssa Bouska, Waseem Lone, et al. Long non coding RNA signatures with diagnostic and prognostic significance in aggressive B-cell lymphoma. Blood 2016;128:1759.

37. Thompson EC, Cobb BS, Sabbattini P, et al. Ikaros DNA-binding proteins as integral components of B cell developmental-stage-specific regulatory circuits. Immunity. 2007;26:335-44.

38. Mallampati S, Sun B, Lu Y, et al. Integrated genetic approaches identify the molecular mechanisms of Sox4 in early B-cell development: intricate roles for RAG1/2 and CK1 $\varepsilon$. Blood. 2014;123:4064-76.

39. Yoshitomi H, Kobayashi S, Miyagawa-Hayashino A, et al. Human Sox4 facilitates the development of CXCL13-producing helper T cells in inflammatory environments. Nat Commun. 2018:9:3762

40. Chen JQ, Papp G, Póliska S, et al. MicroRNA expression profiles identify disease-specific alterations in systemic lupus erythematosus and primary Sjögren's syndrome. PLoS One. 2017;12:e0174585.

41. Kim C, Hu B, Jadhav RR, et al. Activation of miR-21-regulated pathways in immune aging selects against signatures characteristic of memory $T$ cells. Cell Rep. 2018;25:2148-62 e5.

\section{Publisher's Note}

Springer Nature remains neutral with regard to jurisdictional claims in published maps and institutional affiliations.

Ready to submit your research? Choose BMC and benefit from:

- fast, convenient online submission

- thorough peer review by experienced researchers in your field

- rapid publication on acceptance

- support for research data, including large and complex data types

- gold Open Access which fosters wider collaboration and increased citations

- maximum visibility for your research: over $100 \mathrm{M}$ website views per year

At BMC, research is always in progress.

Learn more biomedcentral.com/submissions 